\title{
An application of grand Furuta inequality to a type of operator equation
}

\author{
Jian Shi \\ College of Mathematics and Computer Science, Hebei University, Baoding 071002, Hebei, China \\ E-mail: mathematic@126.com
}

Copyright (C2014 Jian Shi. This is an open access article distributed under the Creative Commons Attribution License, which permits unrestricted use, distribution, and reproduction in any medium, provided the original work is properly cited.

\begin{abstract}
The existence of positive semidefinite solutions of the operator equation $\sum_{j=1}^{n} A^{n-j} X A^{j-1}=Y$ is investigated by applying grand Furuta inequality. If there exists positive semidefinite solutions of the operator equation, one of the special types of $Y$ is obtained, which extends the related result before. Finally, an example is given based on our result.
\end{abstract}

Keywords: grand Furuta inequality, operator equation, matrix equation, positive semidefinite operator.

\section{Introduction}

A capital letter ( $\operatorname{such}$ as $T$ ) means a bounded linear operator on a Hilbert space. $T \geqslant 0$ and $T>0$ mean a positive semidefinite operator and a positive definite operator, respectively.

In the middle of last century, E. Heinz et al. studied operator theory and obtained the following famous theorem: Theorem 1.1 (Löwner-Heinz Inequality, [16] [13]). If $A \geqslant B \geqslant 0$, then $A^{\alpha} \geqslant B^{\alpha}$ holds for any $\alpha \in[0,1]$.

It is essential to notice that Löwner-Heinz inequality does not always hold for $\alpha>1$.

In 1987, T. Furuta proved the following result which is an important and historical extension of Löwner-Heinz inequality:

Theorem 1.2 (Furuta Inequality, [8]). If $A \geqslant B \geqslant 0$, then for each $r \geqslant 0$,

$$
\begin{aligned}
& \left(B^{\frac{r}{2}} A^{p} B^{\frac{r}{2}}\right)^{\frac{1}{q}} \geqslant\left(B^{\frac{r}{2}} B^{p} B^{\frac{r}{2}}\right)^{\frac{1}{q}}, \\
& \left(A^{\frac{r}{2}} A^{p} A^{\frac{r}{2}}\right)^{\frac{1}{q}} \geqslant\left(A^{\frac{r}{2}} B^{p} A^{\frac{r}{2}}\right)^{\frac{1}{q}}
\end{aligned}
$$

hold for $p \geqslant 0, q \geqslant 1$ with $(1+r) q \geqslant p+r$.

Afterwards, the studies of the theory of operator inequalities have been developed quickly and some results related to Furuta inequality have been obtained in recent twenty-five years, such as $[1,2,9,17,23,24,25]$. It is well known that Furuta inequality has many applications. See [3, 5, 11, 14, 15, 20, 21, 22, 26].

In 1995, T. Furuta showed another operator inequality which interpolates Furuta inequality: 
Theorem 1.3 (Grand Furuta Inequality, [9]). If $A \geqslant B \geqslant 0$ with $A>0$, then for each $t \in[0,1]$ and $p \geqslant 1$,

$$
A^{1-t+r} \geqslant\left\{A^{\frac{r}{2}}\left(A^{-\frac{t}{2}} B^{p} A^{-\frac{t}{2}}\right)^{s} A^{\frac{r}{2}}\right\}^{\frac{1-t+r}{(p-t) s+r}}
$$

holds for $s \geqslant 1$ and $r \geqslant t$.

Consequently, some nice proofs of grand Furuta inequality were shown, such as [6] and [10]. K. Tanahashi, in [18], proved that the outer exponent value of (1.3) is the best possible. Later on, the proof was improved by T. Yamazaki and M. Fujii et al. in [19] and [7], respectively.

Recently, T. Furuta proved the following theorem by Furuta inequality:

Theorem 1.4 ([12]). Let $m$ and $n$ be nature numbers. If $A$ and $B$ are a positive definite operator and a positive semidefinite operator, respectively, then there exists positive semidefinite operator solution $X$ satisfying the following operator equation:

$$
\sum_{j=1}^{n} A^{n-j} X A^{j-1}=A^{\frac{n r}{2(m+r)}}\left(\sum_{i=1}^{m} A^{\frac{n(m-i)}{m+r}} B A^{\frac{n(i-1)}{m+r}}\right) A^{\frac{n r}{2(m+r)}}
$$

for $r$ such that $\begin{cases}r \geqslant 0, & \text { if } n \geqslant m ; \\ r \geqslant \frac{m-n}{n-1}, & \text { if } m \geqslant n \geqslant 2 .\end{cases}$

Our purpose of the present article is to study the existence of positive semidefinite solution of operator equation $\sum_{j=1}^{n} A^{n-j} X A^{j-1}=Y$ by grand Furuta inequality, and show a more generalized special type of $Y$ than Theorem 1.4. Although we use the same method as in [12], we think that careful argument is required, and a more generalized example, especially the expression of $Y$, is also required. Therefore, we have this article.

\section{Positive semidefinite solutions of an operator equation}

Let us recall a useful lemma first.

Lemma 2.1 ([4], [12]). Let $A$ be a positive definite operator and $B$ be a positive semidefinite operator. Let $m$ be a positive integer and $x \geqslant 0$, then

$$
\left.\frac{d}{d x}\left[(A+x B)^{m}\right]\right|_{x=0}=\sum_{j=1}^{m} A^{m-j} B A^{j-1} .
$$

Now we give the main result as follows,

Theorem 2.1. Let $m, n$ and $k$ be positive integers. If $A$ and $B$ are a positive definite operator and a positive semidefinite operator, respectively, then for each $t \in[0,1]$, there exists positive semidefinite operator solution $X$ which satisfies the following operator equation:

$$
\begin{aligned}
& \sum_{j=1}^{n} A^{n-j} X A^{j-1} \\
= & A^{\frac{n r}{2[(m-t) k+r]}}\left(\sum_{i=1}^{k} \sum_{j=1}^{m} A^{\frac{n[2(m-t)(k-i)-t+2(m-j)]}{2[(m-t) k+r]}} B A^{\frac{n[2(j-1)-t+2(m-t)(i-1)]}{2[(m-t) k+r]}}\right) A^{\frac{n r}{2[(m-t) k+r}}
\end{aligned}
$$

for $r$ such that $\begin{cases}r \geqslant t, & \text { if }(1-t) n \geqslant(m-t) k ; \\ r \geqslant \max \left\{\frac{(m-t) k-(1-t) n}{n-1}, t\right\}, & \text { if }(m-t) k \geqslant(1-t) n \text { with } n \geqslant 2 .\end{cases}$

Proof. First, by $A+x B \geqslant A>0$ holds for any $x \geqslant 0$, then $A^{-1} \geqslant(A+x B)^{-1}>0$. Replacing $A$ by $A^{-1}, B$ by $(A+x B)^{-1}, p$ by $m, s$ by $k$ in (1.3), and taking reverse, we have

$$
\left(A^{\frac{r}{2}}\left(A^{-\frac{t}{2}}(A+x B)^{m} A^{-\frac{t}{2}}\right)^{k} A^{\frac{r}{2}}\right)^{\frac{1-t+r}{(m-t) k+r}} \geqslant A^{1-t+r} .
$$


For any $\alpha \in[0,1]$, applying Löwner-Heinz inequality to (2.2), and taking an integer $n$ such that $\frac{1}{n}=\frac{1-t+r}{(m-t) k+r} \cdot \alpha$, then the following inequality is obtained:

$$
\left(A^{\frac{r}{2}}\left(A^{-\frac{t}{2}}(A+x B)^{m} A^{-\frac{t}{2}}\right)^{k} A^{\frac{r}{2}}\right)^{\frac{1}{n}} \geqslant A^{\frac{(m-t) k+r}{n}} .
$$

By $\alpha \in[0,1]$ and the condition of $r$ in grand Furuta inequality, we have to take $r \geqslant t$ if $(1-t) n \geqslant(m-t) k$, or $r \geqslant \max \left\{\frac{(m-t) k-(1-t) n}{n-1}, t\right\}$ if $(m-t) k \geqslant(1-t) n$ with $n \geqslant 2$.

Put $Y(x)=\left(A^{\frac{r}{2}}\left(A^{-\frac{t}{2}}(A+x B)^{m} A^{-\frac{t}{2}}\right)^{k} A^{\frac{r}{2}}\right)^{\frac{1}{n}}$. According to (2.3), we have $Y(x) \geqslant Y(0)=A^{\frac{(m-t) k+r}{n}}$ for any $x \geqslant 0$. Thus, $Y^{\prime}(0) \geqslant 0$. Differentiating $Y^{n}(x)=A^{\frac{r}{2}}\left(A^{-\frac{t}{2}}(A+x B)^{m} A^{-\frac{t}{2}}\right)^{k} A^{\frac{r}{2}}$, using Lemma 2.1, and taking $x=0$, the following equality holds.

$$
\begin{aligned}
& \left.\frac{d}{d x}\left[Y^{n}(x)\right]\right|_{x=0}=\sum_{j=1}^{n} Y(0)^{n-j} Y^{\prime}(0) Y(0)^{j-1} \\
= & \left.\frac{d}{d x}\left[A^{\frac{r}{2}}\left(A^{-\frac{t}{2}}(A+x B)^{m} A^{-\frac{t}{2}}\right)^{k} A^{\frac{r}{2}}\right]\right|_{x=0} \\
= & A^{\frac{r}{2}}\left\{\sum_{i=1}^{k}\left[\left.\left(A^{-\frac{t}{2}}(A+x B)^{m} A^{-\frac{t}{2}}\right)^{k-i}\right|_{x=0}\right] \cdot\left[\left.\left(A^{-\frac{t}{2}}(A+x B)^{m} A^{-\frac{t}{2}}\right)^{\prime}\right|_{x=0}\right]\right. \\
& \left.\cdot\left[\left.\left(A^{-\frac{t}{2}}(A+x B)^{m} A^{-\frac{t}{2}}\right)^{i-1}\right|_{x=0}\right]\right\} A^{\frac{r}{2}} \\
= & A^{\frac{r}{2}}\left\{\sum_{i=1}^{k}\left[A^{(m-t)(k-i)}\left(A^{-\frac{t}{2}}\left(\sum_{j=1}^{m} A^{m-j} B A^{j-1}\right) A^{-\frac{t}{2}}\right) A^{(m-t)(i-1)}\right]\right\} A^{\frac{r}{2}} \\
= & A^{\frac{r}{2}}\left(\sum_{i=1}^{k} \sum_{j=1}^{m} A^{(m-t)(k-i)-\frac{t}{2}+(m-j)} B A^{(j-1)-\frac{t}{2}+(m-t)(i-1)}\right) A^{\frac{r}{2}} .
\end{aligned}
$$

Replacing $Y(0)$ by $A^{\frac{(m-t) k+r}{n}}, Y^{\prime}(0)$ by $X$, we have

$$
\begin{aligned}
& \sum_{j=1}^{n} A^{\frac{(m-t) k+r}{n}(n-j)} X A^{\frac{(m-t) k+r}{n}(j-1)} \\
= & A^{\frac{r}{2}}\left(\sum_{i=1}^{k} \sum_{j=1}^{m} A^{(m-t)(k-i)-\frac{t}{2}+(m-j)} B A^{(j-1)-\frac{t}{2}+(m-t)(i-1)}\right) A^{\frac{r}{2}} .
\end{aligned}
$$

Replacing $A$ by $A^{\frac{n}{(m-t) k+r}}$ in (2.4), (2.1) is obtained.

Remark 2.1. If we take $t=0$ and $k=1$ in Theorem 2.1, the theorem is just Theorem 1.4, which is the main result of $[12]$.

Remark 2.2. According to the related result before, if $A$ and $Y$ are positive semidefinite matrices in matrix equation $\sum_{j=1}^{n} A^{n-j} X A^{j-1}=Y$, then $X$ is also a positive semidefinite matrix, see [4]. However, by Theorem 2.1, in some special cases, if $Y$ can be expressed as the right hand of (2.1) without being a positive semidefinite matrix, there still exists positive semidefinite solution satisfying the matrix equation $\sum_{j=1}^{n} A^{n-j} X A^{j-1}=Y$.

For example, let

$$
A=\left(\begin{array}{cc}
1 & 0 \\
0 & 2 \times 2^{\frac{1}{3}}
\end{array}\right), Y=\left(\begin{array}{cc}
4 & 3 \times 2^{\frac{1}{4}}+6 \times 2^{\frac{3}{4}} \\
3 \times 2^{\frac{1}{4}}+6 \times 2^{\frac{3}{4}} & 32
\end{array}\right) .
$$

Although $Y$ is not a positive semidefinite matrix (because its eigenvalues are $\{37.5589 \ldots,-1.5589 \ldots\}$ ), by simple calculation, the solution of the following matrix equation

$$
A^{2} X+A X A+X A^{2}=Y
$$


is

$$
X=\left(\begin{array}{cc}
\frac{4}{3} & \frac{3 \times 2^{\frac{1}{4}}+6 \times 2^{\frac{3}{4}}}{1+2 \times 2^{\frac{1}{3}}+4 \times 2^{\frac{2}{3}}} \\
\frac{3 \times 2^{\frac{1}{4}}+6 \times 2^{\frac{3}{4}}}{1+2 \times 2^{\frac{1}{3}}+4 \times 2^{\frac{2}{3}}} & \frac{4 \times 2^{\frac{1}{3}}}{3}
\end{array}\right),
$$

which is still a definite matrix whose eigenvalues are $\{2.9013 \ldots, 0.1119 \ldots\}$. The critical reason is that $Y$ can be expressed as follows,

$$
Y=A^{\frac{3}{8}}\left(\sum_{i=1}^{2} \sum_{j=1}^{2} A^{\frac{3\left[3(2-i)-\frac{1}{2}+2(2-j)\right]}{8}} B A^{\frac{3\left[2(j-1)-\frac{1}{2}+3(i-1)\right]}{8}}\right) A^{\frac{3}{8}},
$$

which is the right hand of (2.1) under the condition of $m=2, n=3, k=2, t=\frac{1}{2}, r=1$ and $B=\left(\begin{array}{ll}1 & 1 \\ 1 & 1\end{array}\right)$.

\section{References}

[1] T. Ando, On some operator inequalities, Math. Ann. 279 (1987), 157-159.

[2] T. Ando and F. Hiai, Log majorization and complementary Golden-Thompson type inequalities, Linera Algebra Appl. 197 (1994), 113-131.

[3] A. Aluthge, On p-hyponormal operators for $0<p<1$, Integr. Equat. Oper. Th. 13 (1990), 307-315.

[4] R. Bhatia and M. Uchiyama, The operator equation $\sum_{i=0}^{n} A^{n-i} X B^{i}=Y$, Expo. Math. 27 (2009), 251-255.

[5] M. Fujii, T. Furuta and E. Kamei, Furuta's inequality and its application to Ando's theorem, Linear Algebra Appl. 179 (1993), 161-169.

[6] M. Fujii and E. Kamei, Mean theoretic approach to the grand Furuta inequality, Proc. Amer. Math. Soc. 124 (1996), 2751-2756.

[7] M. Fujii, A. Matsumoto and R. Nakamoto, A short proof of the best possibility for the grand Furuta inequality, J. Inequal. Appl. 4 (1999), 339-344.

[8] T. Furuta, $A \geqslant B \geqslant 0$ assures $\left(B^{r} A^{p} B^{r}\right)^{1 / q} \geqslant B^{(p+2 r) / q}$ for $r \geqslant 0, p \geqslant 0, q \geqslant 1$ with $(1+2 r) q \geqslant p+2 r$, Proc. Amer. Math. Soc. 101 (1987), 85-88.

[9] T. Furuta, An extension of the Furuta inequality and Ando-Hiai log-majorization, Linear Algebra Appl. 219 (1995), 139-155.

[10] T. Furuta, Simplified proof of an order preserving operator inequality, Proc. Japan Acad. 74 (1998), 114.

[11] T. Furuta and M. Yanagida, On powers of p-hyponormal and log-hyponormal operators, J. Inequal. Appl. 5 (2000), 367-380.

[12] T. Furuta, Positive semidefinite solutions of the operator equation $\sum_{j=1}^{n} A^{n-j} X A^{j-1}=B$, Linear Algebra Appl. 432 (2010), 949-955.

[13] E. Heinz, Beiträge zur Störungsteorie der Spektralzerlegung, Math. Ann. 123 (1951), 415-438.

[14] M. Ito and T. Yamazaki, Relations between two inequalities $\left(B^{\frac{r}{2}} A^{p} B^{\frac{r}{2}}\right)^{\frac{r}{p+r}} \geq B^{r}$ and $\left(A^{\frac{r}{2}} B^{p} A^{\frac{r}{2}}\right)^{\frac{p}{p+r}} \geq A^{r}$ and their applications, Integr. Equat. Oper. Th. 44 (2002), 442-450.

[15] C.-S. Lin, On operator order and chaotic operator order for two operators, Linear Algebra Appl. 425 (2007), 1-6.

[16] K. Löwner, Über monotone MatrixFunktionen, Math. Z. 38 (1934), 177-216.

[17] K. Tanahashi, Best possibility of the Furuta inequality, Proc. Amer. Math. Soc. 124 (1996), 141-146.

[18] K. Tanahashi, The best possibility of the grand Furuta inequality, Proc. Amer. Math. Soc. 128 (2000), $511-519$.

[19] T. Yamazaki, Simplified proof of Tanahashi's result on the best possibility of generalized Furuta inequality, Math. Inequal. Appl. 2 (1999), 473-477.

[20] C. Yang and H. Dai, An application of Furuta inequality and its best possibility, Applied Mathematics, A Journal of Chinese Universities Series B. 23 (3)(2008), 326-330. 
[21] C. Yang and J. Yuan, Extensions of the results on powers of p-hyponormal and log-hyponormal operators, J. Inequal. Appl. 1 (2006), 1-14.

[22] J. Yuan and Z. Gao, Structure on powers of p-hyponormal and log-hyponormal operators, Integr. Equat. Oper. Th. 59 (2007), 437-448.

[23] J. Yuan and Z. Gao, Classified construction of generalized Furuta type operator functions, Math. Inequal. Appl. 11 (2008), 189-202.

[24] J. Yuan and Z. Gao, Complete form of Furuta inequality, Proc. Amer. Math. Soc. 136 (2008), 2859-2867.

[25] J. Yuan, Classified construction of generalized Furuta type operator functions, II, Math. Inequal. Appl. 13 (2010), 775-784.

[26] J. Yuan, Furuta inequality and q-hyponormal operators, Oper. Matrices 4 (2010), 405-415. 